\title{
Active Vibration Control of an Elevator Car Using Two Rotary Actuators*
}

\author{
Kimihiko NAKANO**, Ryuzo HAYASHI***, Yoshihiro SUDA**, \\ Naoaki NOGUCHI**** and Atsushi ARAKAWA $* * * *$ \\ ** Institute of Industrial Science, The University of Tokyo \\ 4-6-1 Komaba Meguro-ku Tokyo 153-8505 Japan \\ E-mail: knakano@iis.u-tokyo.ac.jp \\ ***Department of Mechanical Systems Engineering, Tokyo University of Agriculture and Technology, \\ 2-24-16 Naka-cho, Koganei, Tokyo, 184-8588 Japan \\ **** Mechanical Engineering Research Laboratory, Hitachi, Ltd, \\ 832-2 Horiguchi, Hitachinaka, Ibaraki 312-0034 Japan
}

\begin{abstract}
A new control device is proposed to isolate an elevator cabin from irregularities on a guide rail. The device consists of two rotary electric motors with disks and eccentric masses. One motor produces torque to rotate the eccentric mass according to the command signals from the controller for horizontal control, while the other rotates the mass to eliminate the unwanted vertical force. To design the optimal controller to reduce the horizontal vibration of the cabin, the dynamics of the cabin, including the proposed control device, are described by a linear equation. The performance of the proposed control system is examined through numerical simulations taking into consideration the nonlinear characteristics of the rotating masses, which are neglected in designing the controller, and through experiments with a miniature elevator cabin carrying the proposed actuator. The results indicate the proposed control device reduces the horizontal vibration without producing the unwanted vertical vibration.
\end{abstract}

Key words: Elevator, Vibration Control, Optimal Control, Vibration Control Device, Dynamic Absorber

\section{Introduction}

In recent years the travelling speeds of elevator cabins have increased significantly, and passengers have become much more aware of the ride quality they experience. Consequently the need to suppress vibrations of the cabin has become an important issue in elevator system design. Some research groups have focused on the analysis of the vibrations caused by the movement of the travelling rope ${ }^{(1)}$, and discussed methods for suppressing these vibrations ${ }^{(2),(3)}$. Active vibration control is a major method for suppressing the vibration. To isolate the cabin from horizontal vibrations caused by the roughness of the rails which guide the elevator cabin, a control system using a servo-motor ${ }^{(4),(5)}$, an active mass damper system ${ }^{(6),(7)}$ and active roller guides ${ }^{(8)}$ were trialed. The performances of these systems were examined and some of them were already being used in practice. However, to make them more acceptable, there is a need to reduce their size so that the engineers do not have to change the design of the typical elevator cabin. This paper proposes a new device for active vibration control to isolate the horizontal vibrations resulting from irregularities of the rails.

*Received 27 July, 2010 (No. T1-08-0831) Japanese Original : Trans. Jpn. Soc. Mech. Eng., Vol.75, No.753, C (2009), pp.1377-1382 (Received 19 Sep., 2008) [DOI: 10.1299/jsdd.5.155]

Copyright $\odot 2011$ by JSME 
The proposed control device consists of two rotary electric motors with eccentric masses. The two masses rotate in opposite directions at the same speed to produce horizontal forces without generating any unwanted vertical forces. Compared to a design using a linear actuator, we expected to make the device more compact ${ }^{(9)}$.

The equations of the motion are formulated assuming the main rope which suspends the elevator cabin is elastic, and the cabin is allowed to move in roll, horizontal and vertical directions. One of the motors produces control torque according to the command signal from the controller, while the other motor rotates to counteract any vertical forces. The controller is designed using an optimal control scheme to suppress both roll and horizontal vibration. The performance of the proposed system is examined both through numerical simulations taking into consideration the nonlinear characteristics of the rotating eccentric masses and through laboratory experiments using a miniature elevator cabin carrying the proposed control device.

\section{Proposed Control Device}

The proposed control device is shown in Fig.1. It is composed of two electric rotary actuators with identical eccentric masses $m_{\mathrm{d}}$. Each mass is attached to a uniform rigid disk whose inertia moment around its center is $I_{\mathrm{d}}$. By rotating the eccentric mass, the rotary actuator produces control force in the lateral direction to reduce vibrations of the cabin; however, it also produces unwanted vertical forces.

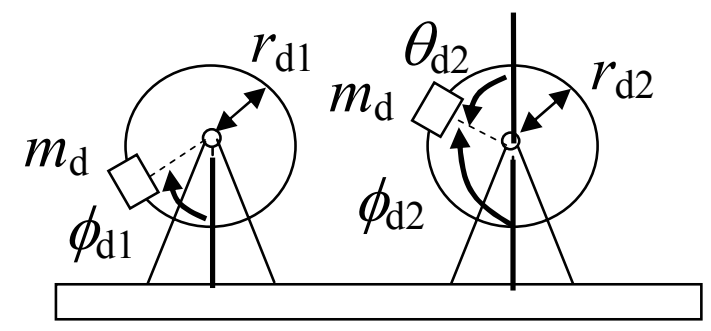

Fig. 1 The proposed vibration control device for the elevator cabin.

To eliminate the unwanted vertical forces the other actuator produces the counter vertical force. It is successful when the rotating angles $\phi_{\mathrm{d} 1}, \phi_{\mathrm{d} 2}$ of the two masses keep the following relationship:

$$
\phi_{d 1}=\pi-\phi_{d 2}
$$

We use the new coordinate for the actuator angles $\theta_{\mathrm{d} 1}, \theta_{\mathrm{d} 2}$. While the coordinate for the first actuator is the same as mentioned above, the initial angle of the second actuator is set to be $\pi$ and the positive direction is reversed, that is:

$$
\begin{aligned}
& \theta_{d 1}=\phi_{d 1} \\
& \theta_{d 2}=\pi-\phi_{d 2}
\end{aligned}
$$

In this new coordinate system, the condition that cancels the vertical forces produced by the two actuators is given by:

$$
\theta_{d 1}=\theta_{d 2}
$$

\section{Equation of Motion}

Figure 2 shows the dynamic model of the elevator cabin with the proposed control device. The roughness of the guide disturbs the cabin. The displacements of the upper and 
lower springs are expressed as $x_{0 \mathrm{u}}$ and $x_{0 \mathrm{~d}}$. When the lateral and vertical displacements and the angle of the center of the gravity of the cabin are defined as $x_{\mathrm{c}}, z_{\mathrm{c}}$, and $\theta_{\mathrm{c}}$, the positions of the eccentric masses $\left(x_{d 1}, z_{d 1}\right)$ and $\left(x_{d 2}, z_{d 2}\right)$ can be expressed as:

$$
\begin{aligned}
& \left(x_{d 1}, z_{d 1}\right)=\left(x_{c}-l_{x d 1}+l_{d} \theta_{c}-r_{d} \sin \theta_{d 1}, \quad z_{c}-l_{d}-r_{d} \cos \theta_{d 1}\right) \\
& \left(x_{d 2}, z_{d 2}\right)=\left(x_{c}+l_{x d 2}+l_{d} \theta_{c}-r_{d} \sin \theta_{d 2}, \quad z_{c}-l_{d}+r_{d} \cos \theta_{d 2}\right)
\end{aligned}
$$

Then the total kinetic energy $T$ and the potential energy $P$ of the system are written as:

$$
\begin{aligned}
T & =\frac{1}{2}\left\{m_{c}\left(\dot{x}_{c}{ }^{2}+\dot{z}_{c}{ }^{2}\right)+I_{c} \dot{\theta}_{c}{ }^{2}+I_{d} \dot{\theta}_{d 1}{ }^{2}+I_{d} \dot{\theta}_{d 2}{ }^{2}+m_{d}\left(\dot{x}_{d 1}{ }^{2}+\dot{z}_{d 1}{ }^{2}\right)+m_{d}\left(\dot{x}_{d 2}{ }^{2}+\dot{z}_{d 2}{ }^{2}\right)\right\} \\
P & =\frac{1}{2}\left\{k_{u}\left(x_{c}-l_{1} \theta_{c}-x_{0 u}\right)^{2}+k_{d}\left(x_{c}+l_{2} \theta_{c}-x_{0 d}\right)^{2}+k_{r} \dot{z}_{c}{ }^{2}\right\}-m_{d} g r_{d} \cos \theta_{d 1}+m_{d} g r_{d} \cos \theta_{d 2}
\end{aligned}
$$

The elevator cabin does not have dampers, but in practice it dissipates energy inside it. Assuming the forces dissipating energy are viscous damping forces, we introduce Rayleigh's dissipation function $R$, written as:

$$
R=\frac{1}{2}\left\{c_{p} \dot{x}_{c}^{2}+c_{c} \dot{\theta}_{c}^{2}+c_{r} \dot{z}_{c}^{2}+c_{d 1} \dot{\theta}_{d 1}^{2}+c_{d 2} \dot{\theta}_{d 2}^{2}\right\}
$$

From Lagrange's equation, the equation of motion of the system is obtained as shown in Eq. 10. In this equation, $\tau_{1}$ and $\tau_{2}$ are torques produced by the Actuators 1 and 2. The equation of motion represented in Eq. 10 includes nonlinear elements; however, it is easier to develop a linear equation in designing a controller. Assuming small displacements and angles, Eq. 10 reduces to a linear equation as shown in Eq. 11. From this equation, we can see the vertical motion has no relationship to any other motion, and therefore we can omit the second row.

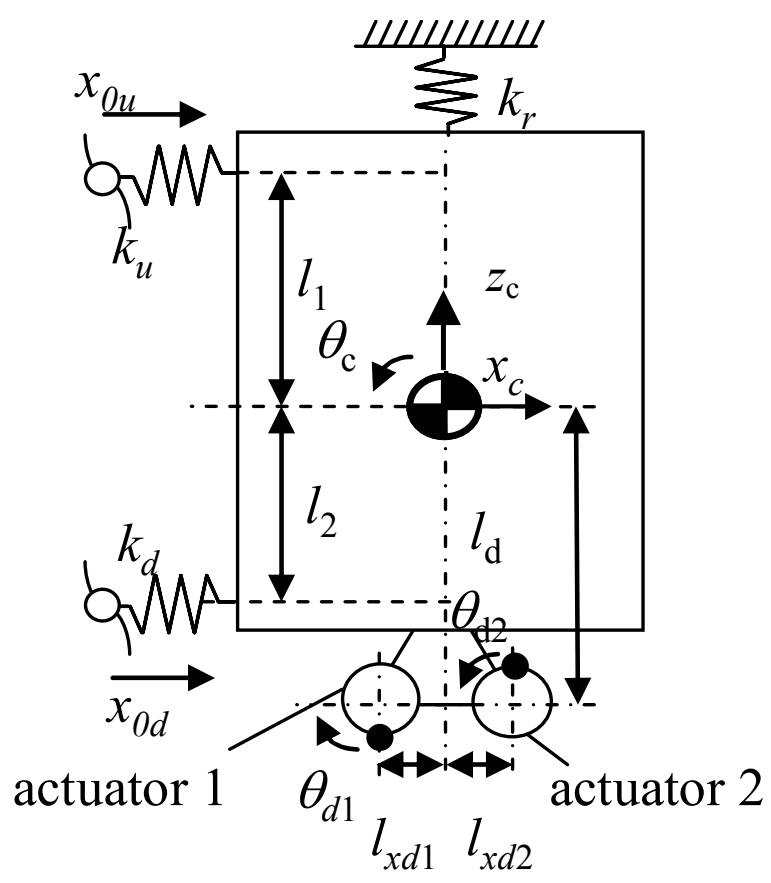

Fig. 2 The elevator cabin with the proposed control device. 


$\left[\begin{array}{ccccc}m_{c}+2 m_{d} & 0 & 2 m_{d} l_{d} & -m_{d} r_{a} \cos \theta_{d 1} & -m_{d} r_{a} \cos \theta_{d 2} \\ 0 & m_{c}+2 m_{d} & 0 & m_{d} r_{a} \sin \theta_{d 1} & -m_{d} r_{a} \sin \theta_{d 2} \\ 2 m_{d} l_{d} & 0 & I_{c}+2 m_{d} l_{d}{ }^{2} & -m_{d} l_{d} r_{a} \cos \theta_{d 1} & -m_{d} l_{d} r_{a} \cos \theta_{d 2} \\ -m_{d} r_{a} \cos \theta_{d 1} & m_{d} r_{a} \sin \theta_{d 1} & -m_{d} l_{d} r_{a} \cos \theta_{d 1} & m_{d} r_{a}{ }^{2}+I_{d} & 0 \\ -m_{d} r_{a} \cos \theta_{d 2} & -m_{d} r_{a} \sin \theta_{d 2} & -m_{d} l_{d} r_{a} \cos \theta_{d 2} \cdot & 0 & m_{d} r_{a}{ }^{2}+I_{d}\end{array}\right]\left[\begin{array}{c}\ddot{x}_{c} \\ \ddot{z}_{c} \\ \ddot{\theta}_{c} \\ \ddot{\theta}_{d 1} \\ \ddot{\theta}_{d 2}\end{array}\right]$
$+\left[\begin{array}{ccccc}k_{u}+k_{d} & 0 & -k_{u} l_{1}+k_{d} l_{2} & 0 & 0 \\ 0 & k_{r} & 0 & 0 & 0 \\ -k_{u} l_{1}+k_{d} l_{2} & 0 & l_{1}{ }^{2} k_{u}+l_{2}{ }^{2} k_{d} & 0 & 0 \\ 0 & 0 & 0 & 0 & 0 \\ 0 & 0 & 0 & 0 & 0\end{array}\right]\left[\begin{array}{c}x_{c} \\ z_{c} \\ \theta_{c} \\ \theta_{d 1} \\ \theta_{d 2}\end{array}\right]+\left[\begin{array}{ccccc}c_{x} & 0 & 0 & 0 & 0 \\ 0 & c_{r} & 0 & 0 & 0 \\ 0 & 0 & c_{\theta} & 0 & 0 \\ 0 & 0 & 0 & c_{d} & 0 \\ 0 & 0 & 0 & 0 & c_{d}\end{array}\right]\left[\begin{array}{c}\dot{x}_{c} \\ \dot{z}_{c} \\ \dot{\theta}_{c} \\ \dot{\theta}_{d 1} \\ \dot{\theta}_{d 2}\end{array}\right]$ $+\left[\begin{array}{c}m_{d} r_{a} \dot{\theta}_{d 1}{ }^{2} \sin \theta_{d 1}+m_{d} r_{a} \dot{\theta}_{d 2}{ }^{2} \sin \theta_{d 2} \\ m_{d} r_{a} \dot{\theta}_{d 1}{ }^{2} \cos \theta_{d 1}-m_{d} r_{a} \dot{\theta}_{d 2}{ }^{2} \cos \theta_{d 2} \\ m_{d} l_{d} r_{a} \dot{\theta}_{d 1}{ }^{2} \sin \theta_{d 1}+m_{d} l_{d} r_{a} \dot{\theta}_{d 2}{ }^{2} \sin \theta_{d 2} \\ m_{d} g r_{a} \sin \theta_{d 1} \\ -m_{d} g r_{a} \sin \theta_{d 2}\end{array}\right]=\left[\begin{array}{cc}k_{u} & k_{d} \\ 0 & 0 \\ -l_{1} k_{u} & l_{2} k_{d} \\ 0 & 0 \\ 0 & 0\end{array}\right]\left[\begin{array}{l}x_{0 u} \\ x_{0 d}\end{array}\right]+\left[\begin{array}{ll}0 & 0 \\ 0 & 0 \\ 0 & 0 \\ 1 & 0 \\ 0 & 1\end{array}\right]\left[\begin{array}{l}\tau_{1} \\ \tau_{2}\end{array}\right]$

$\left[\begin{array}{ccccc}m_{c}+2 m_{d} & 0 & 2 m_{d} l_{d} & -m_{d} r_{a} & -m_{d} r_{a} \\ 0 & m_{c}+2 m_{d} & 0 & 0 & 0 \\ 2 m_{d} l_{d} & 0 & I_{c}+2 m_{d} l_{d}{ }^{2} & -m_{d} l_{d} r_{a} & -m_{d} l_{d} r_{a} \\ -m_{d} r_{a} & 0 & -m_{d} l_{d} r_{a} & m_{d} r_{a}{ }^{2}+I_{d} & 0 \\ -m_{d} r_{a} & 0 & -m_{d} l_{d} r_{a} & 0 & m_{d} r_{a}{ }^{2}+I_{d}\end{array}\right]\left[\begin{array}{c}\ddot{x}_{c} \\ \ddot{z}_{c} \\ \ddot{\theta}_{c} \\ \ddot{\theta}_{d 1} \\ \ddot{\theta}_{d 2}\end{array}\right]+\left[\begin{array}{ccccc}c_{x} & 0 & 0 & 0 & 0 \\ 0 & c_{r} & 0 & 0 & 0 \\ 0 & 0 & c_{\theta} & 0 & 0 \\ 0 & 0 & 0 & c_{d} & 0 \\ 0 & 0 & 0 & 0 & c_{d}\end{array}\right]\left[\begin{array}{c}\dot{x}_{c} \\ \dot{z}_{c} \\ \dot{\theta}_{c} \\ \dot{\theta}_{d} \\ \dot{\theta}_{d 2}\end{array}\right]$
$+\left[\begin{array}{ccccc}k_{u}+k_{d} & 0 & -k_{u} l_{1}+k_{d} l_{2} & 0 & 0 \\ 0 & k_{r} & 0 & 0 & 0 \\ -k_{u} l_{1}+k_{d} l_{2} & 0 & l_{1}{ }^{2} k_{u}+l_{2}{ }^{2} k_{d} & 0 & 0 \\ 0 & 0 & 0 & m_{d} g r_{a} & 0 \\ 0 & 0 & 0 & 0 & -m_{d} g r_{a}\end{array}\right]\left[\begin{array}{c}x_{c} \\ z_{c} \\ \theta_{c} \\ \theta_{d 1} \\ \theta_{d 2}\end{array}\right]=\left[\begin{array}{cc}k_{u} & k_{d} \\ 0 & 0 \\ -l_{1} k_{u} & l_{2} k_{d} \\ 0 & 0 \\ 0 & 0\end{array}\right]\left[\begin{array}{cc}0 \\ 0 & 0 \\ x_{0 u} \\ x_{0 d}\end{array}\right]+\left[\begin{array}{cc}0 \\ 0 \\ 1 & 0 \\ 0 & 1\end{array}\right]\left[\begin{array}{c}\tau_{1} \\ \tau_{2}\end{array}\right]$

To maintain $\theta_{d l}=\theta_{d 2}$, the equations of eccentric masses (the equations of the fourth and fifth rows) need be identical. Then the torque $\tau_{2}$ should be:

$$
\tau_{2}=\tau_{1}-m_{d} g r_{a} \theta_{d 1}-m_{d} g r_{a} \theta_{d 2}
$$

When this relationship is maintained, the fifth row can be omitted. It is not easy to produce such a torque, since a practical system includes modelling error. Therefore it is better to design a regulator such as a PD controller to control $\theta_{\mathrm{d} 2}$ to follow $\theta_{\mathrm{d} 1}$. However the model used in the numerical simulation assumes an ideal system, for which there are no modelling errors, and there is complete control of $\theta_{\mathrm{d} 2}$. This condition is identical for the torque produced by Actuator 2 as shown in Eq. 12. Then the equation of motion reduces to the following simple linear equation:

$$
\begin{aligned}
& {\left[\begin{array}{ccc}
m_{c}+2 m_{d} & 2 m_{d} l_{d} & -2 m_{d} r_{a} \\
2 m_{d} z_{2} & I_{c}+2 m_{d} l_{d}{ }^{2} & -2 m_{d} l_{d} r_{a} \\
-2 m_{d} r_{a} & -2 m_{d} z_{2} r_{a} & 2 m_{d} r_{a}{ }^{2}+2 I_{d}
\end{array}\right]\left[\begin{array}{c}
\ddot{x}_{c} \\
\ddot{\theta}_{c} \\
\ddot{\theta}_{d 1}
\end{array}\right]+\left[\begin{array}{ccc}
c_{x} & 0 & 0 \\
0 & c_{r} & 0 \\
0 & 0 & 2 c_{d}
\end{array}\right]\left[\begin{array}{c}
\dot{x}_{c} \\
\dot{\theta}_{c} \\
\dot{\theta}_{d 1}
\end{array}\right]} \\
& +\left[\begin{array}{ccc}
k_{u}+k_{d} & -k_{u} l_{1}+k_{d} l_{2} & 0 \\
-k_{u} z_{1}+k_{d} z_{2} & z_{1}{ }^{2} k_{u}+z_{2}{ }^{2} k_{d} & 0 \\
0 & 0 & 2 m_{d} g r_{a}
\end{array}\right]\left[\begin{array}{c}
x_{c} \\
\theta_{c} \\
\theta_{d 1}
\end{array}\right]=\left[\begin{array}{ccc}
k_{u} & k_{d} \\
-z_{1} k_{u} & z_{2} k_{d} \\
0 & 0
\end{array}\right]\left[\begin{array}{l}
x_{0 u} \\
x_{0 d}
\end{array}\right]+\left[\begin{array}{l}
0 \\
0 \\
2
\end{array}\right]
\end{aligned}
$$

The values of the parameters utilized in this study are shown in Table 1. 
Table 1 Values of the parameters

\begin{tabular}{lll}
\hline Description & Symbols & Values \\
\hline Damping coefficients & $c_{d}$ & $1.2 \mathrm{mNm} /(\mathrm{rad} / \mathrm{s})$ \\
& $c_{\mathrm{r}}, c_{\mathrm{x}}$ & $29.0,41.1 \mathrm{~N} /(\mathrm{m} / \mathrm{s})$ \\
& $c_{\theta}$ & $3.58 \mathrm{Nm} /(\mathrm{rad} / \mathrm{s})$ \\
Gravity & $g$ & $9.8 \mathrm{~m} / \mathrm{s}^{2}$ \\
Spring constants & $k_{\mathrm{d}}, k_{\mathrm{r}}, k_{\mathrm{u}}$ & $1424,13265,1424 \mathrm{~N} / \mathrm{m}$ \\
Masses & $m_{\mathrm{c},} m_{\mathrm{d}}$ & $21,0.2 \mathrm{~kg}$ \\
Radius & $r_{\mathrm{d}}$ & $0.05 \mathrm{~m}$ \\
Length & $l_{1}, l_{2}, l_{\mathrm{d}}$ & $0.415,0.230,0.195 \mathrm{~m}$ \\
Inertia moment & $I_{y y}, I_{d}$ & $1.38 \mathrm{kgm}^{2}, 0.52 \mathrm{gm}^{2}$ \\
\hline
\end{tabular}

\section{Design of a Controller}

An optimal control scheme is applied to the design of the feedback controller. The main purpose of the active control is to reduce horizontal and roll acceleration, therefore, with trial and error, the cost function is obtained as:

$$
J=\int_{0}^{\infty}\left(X^{T} Q X+u^{T} R u\right) d t
$$

where

$$
\begin{aligned}
& Q=\left[\begin{array}{cccccc}
4 \times 10^{6} & 0 & 0 & 0 & 0 & 0 \\
0 & 2.5 \times 10^{5} & 0 & 0 & 0 & 0 \\
0 & 0 & 0.1 & 0 & 0 & 0 \\
0 & 0 & 0 & 0.1 & 0 & 0 \\
0 & 0 & 0 & 0 & 0.1 & 0 \\
0 & 0 & 0 & 0 & 0 & 0.1
\end{array}\right] \\
& R=[1] \\
& X=\left[\begin{array}{llllll}
x_{c} & \theta_{c} & \theta_{d 1} & \dot{x}_{c} & \dot{\theta}_{c} & \dot{\theta}_{d 1}
\end{array}\right]^{T}
\end{aligned}
$$

The values of matrixes $Q$ and $R$ are selected to reduce the amplitude of vibration to less than a half at the first mode. We define the in-phase disturbance as $x_{0 \text { in }}$ and the reverse-phase disturbance as $x_{0 \mathrm{re}}$ which can be expressed by:

$$
\begin{aligned}
& x_{0 i n}=\frac{x_{0 u}+x_{0 d}}{2} \\
& x_{0 r e}=\frac{x_{0 u}-x_{0 d}}{2}
\end{aligned}
$$

Figure 3 presents the frequency responses from in-phase or reverse-phase disturbances to lateral displacement or roll, where 'with control' indicates the elevator cabin for which the proposed control device is installed and operated, while 'before install' represents the cabin where the device is not installed. First and second mode natural frequencies are $1.7 \mathrm{~Hz}$ and $2.3 \mathrm{~Hz}$. This control device is effective in suppressing the gains especially for the first mode, which is considered to have the most influence on the ride quality. Improving the performance for the second and the higher mode is our future topic.

Numerical simulations using the fourth order Runge-Kutta method are carried out with the cabin exposed to a sinusoidal in-phase disturbance whose amplitude is $0.4 \mathrm{~mm}$ and frequency is $1.7 \mathrm{~Hz}$. In this simulation, the non-linear elements are considered, that is, the equation shown in Eq. 10 is used. Figure 4 shows the results. The amplitudes of the horizontal and rotating vibrations of the controlled system are reduced by approximately 
one third and one half compared with those of the system without control. However, when the system is controlled, a vertical acceleration is produced, which is not found when the system is uncontrolled, but its amplitude is less than $10^{-7} \mathrm{~m} / \mathrm{s}^{2}$, so that its effect is negligible.

(a)

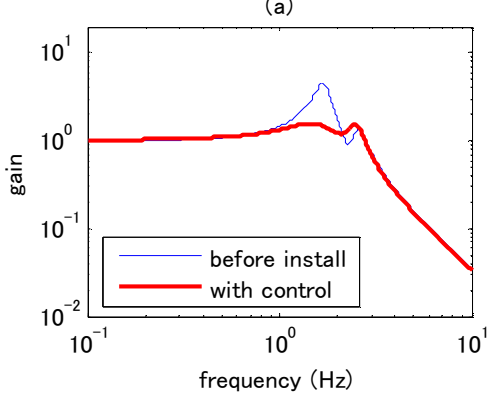

(b)

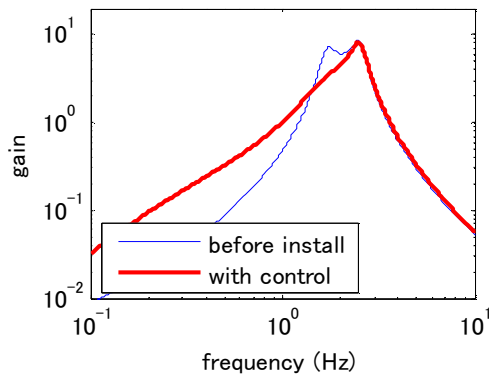

(c)

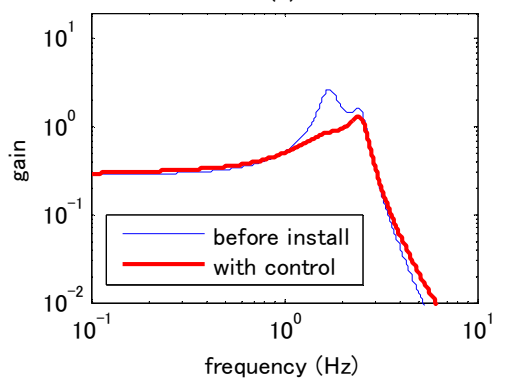

(d)

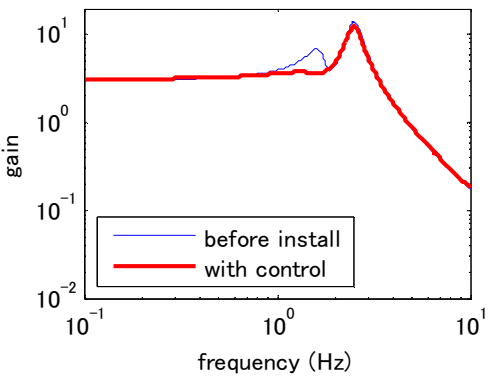

Fig. 3 Frequency responses, which are the gains (a) from the in-phase disturbance to the lateral displacement, (b) from the in-phase disturbance to the roll, (c) from the reverse-phase disturbance to the lateral displacement, and (d) from the reverse-phase disturbance to the roll.
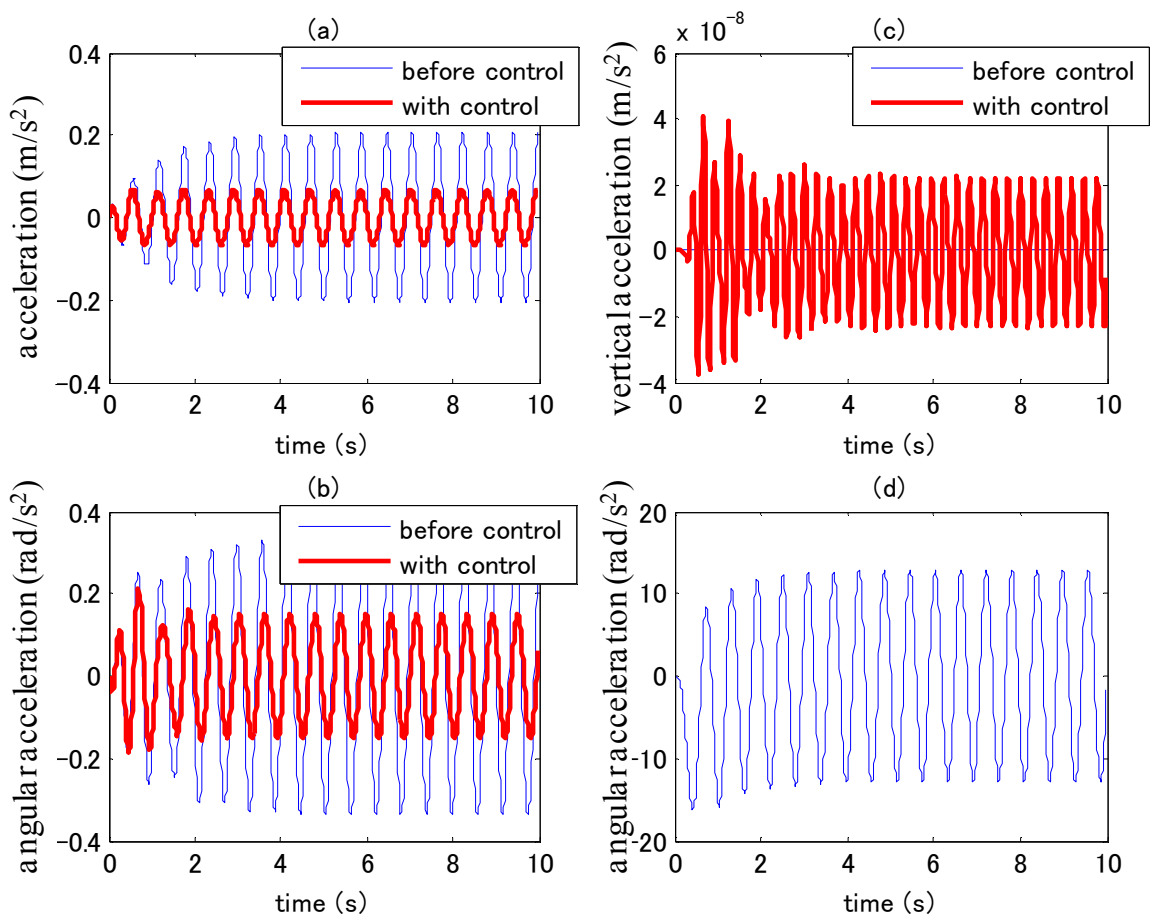

Fig. 4 The results of the numerical simulation under the same condition as the experiments, where (a) represents the lateral acceleration at the center of gravity, (b) represents the roll acceleration, (c) represents the vertical acceleration of the cabin and (d) represents the rotating angle of the disk of Actuator 1. 


\section{Experimental setup}

Figures 5 and 6 show a photograph of the experimental setup and its schematic view. The miniature cabin with the proposed control device is inside the frame, which is shaken by the vibration exciter in the horizontal direction, and is allowed to move in the vertical and the horizontal directions and rotate around the center of gravity. There are four springs, which connect the upper and lower parts of the cabin horizontally to the frame, and an axis for rotation at the center of gravity, which is supported by four springs in the vertical direction.

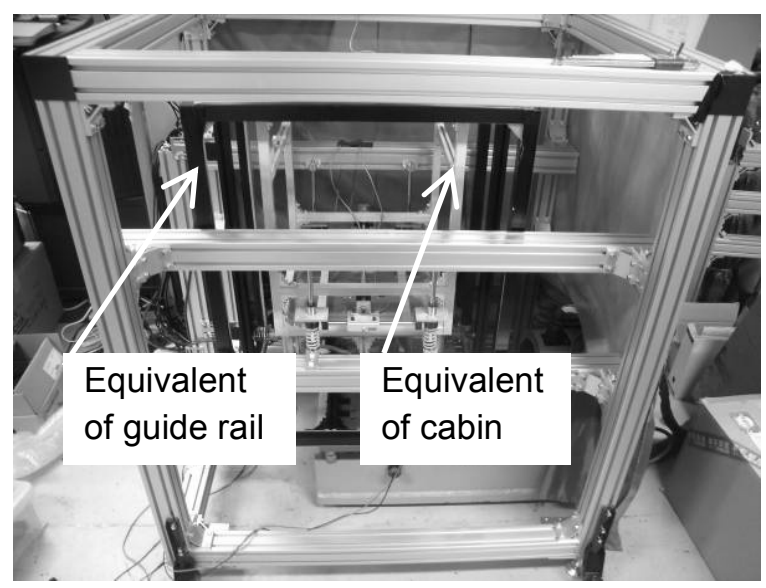

Fig. 5 A photograph of the experimental setup.

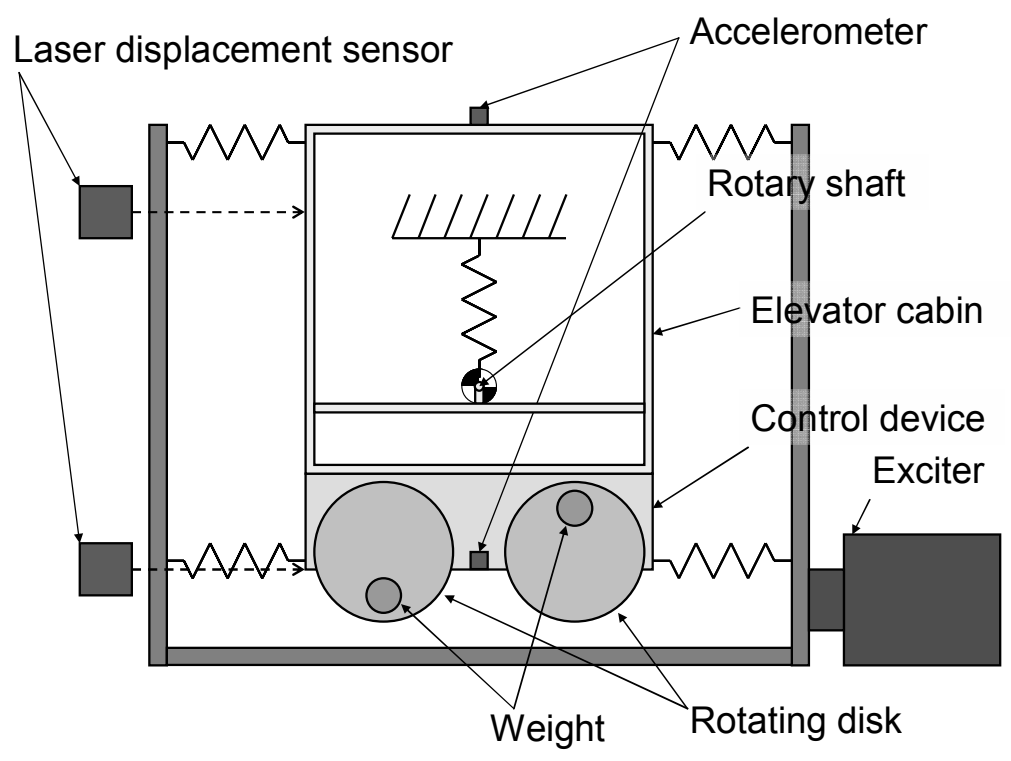

Fig. 6 A schematic view of the experimental setup.

Two laser displacement sensors and two accelerometers measure the displacements and accelerations at the upper and the lower parts of the cabin. The horizontal and rotating angles of the cabin are estimated from the values measured by these sensors. Another displacement sensor and accelerometer are installed to measure the displacement, and the acceleration of the table of the exciter. The control device has two disks with weights, which are rotated by electric motors. Its rotating angles are measured by encoders installed on the motor axes. Measured data are transmitted to a digital signal processing unit and command signals are sent to two electric motors to control the vibration of the cabin. In the 
experiments, the rotating angle of the second actuator was controlled with PID controller to keep the relationship shown in Eq. 4. We then applied the controller, which was designed using the reduced order model written in Eq. 13, to the experimental system.

\section{Results}

Figure 7 shows the results of the experiment when using the in-phase sinusoidal displacement whose amplitude is $0.4 \mathrm{~mm}$ and frequency is $1.7 \mathrm{~Hz}$. In this figure 'without control' indicates the system where the proposed control device is installed but not operated; and the rotating disks are fixed. The lateral and the roll accelerations are reduced to less than the half of those without control. Theoretically vertical vibration is not caused by the lateral vibration in the system 'without control'; therefore the measured acceleration is due to factors not considered in the equation of motion. The amplitudes of the vertical accelerations seem to be the same between the experiment and the numerical simulation. This indicates the proposed device does not cause the significant unwanted vertical vibration. These results show the proposed method is effective for suppression of vibration of the elevator cabin under these experimental conditions.

The absolute values of the experimental results are approximately half those of the numerical simulations. This is due to errors in the identification of the coefficient of internal damping. The internal damping forces mainly originate from the friction forces, therefore it is difficult to identify it as viscous damping. Since the ratio of the vibration amplitude of the controlled to the uncontrolled systems is similar in the experiment and the numerical simulation, it is concluded that the isolation performance can be examined using this model.

(a)

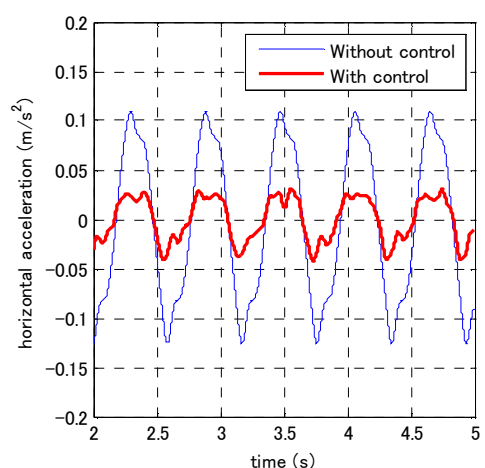

(b)

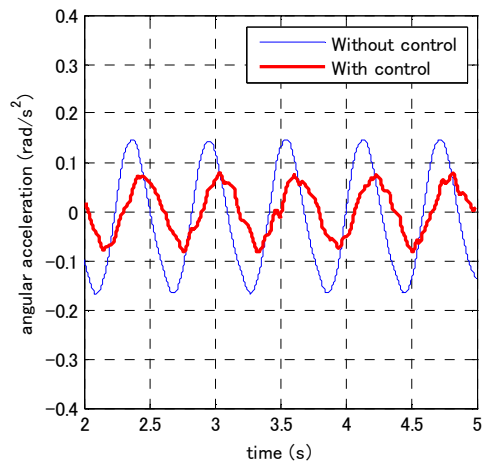

(c)

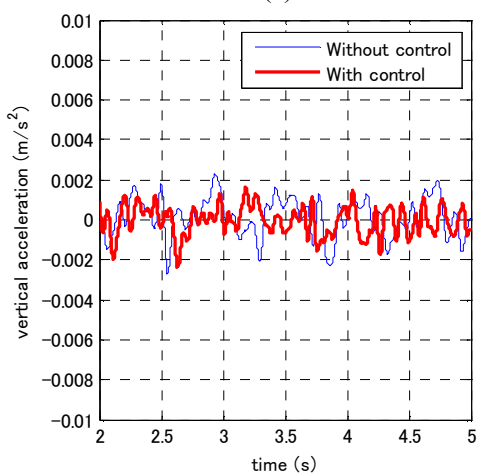

(d)

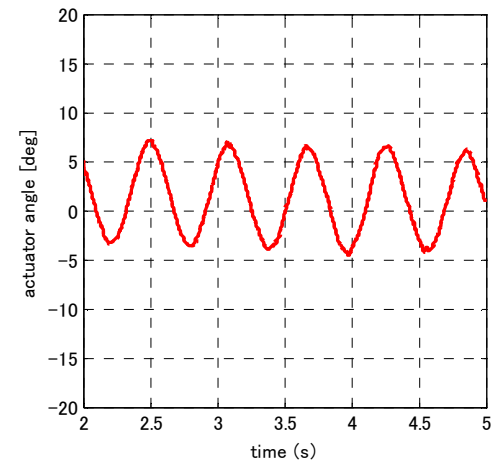

Fig. 7 Results of the experiment when the amplitude and the frequency of the in-phase sinusoidal displacement are $0.4 \mathrm{~mm}$ and $1.7 \mathrm{~Hz}$; (a) represents the lateral acceleration at the center of gravity, (b) represents the roll acceleration, (c) represents the vertical acceleration of the cabin and (d) represents the rotating angle of the disk of the Actuator 1. 


\section{Conclusions}

The authors proposed a rotary actuator system to suppress the vibration of the cabin of the elevator. The controller is designed using optimal control theory and its performance is examined through experiments and numerical simulations. In summary, the following observations are made:

1. The proposed control device reduces the vibration, especially in the first mode.

2. The proposed control device provides good isolation from the in-phase vibration in the first mode.

3. The proposed model for the numerical simulation is effective in examining the isolation performance through experiments and numerical simulation.

4. The proposed control device does not increase the vertical vibration.

\section{References}

(1) Zhu W.D., Teppo L.J.: Design and analysis of a scaled model of a high-rise, high-speed elevator, Journal of Sound and Vibration, 264, (2003), pp.707-731.

(2) R.-F. Fung, J.-H. Lin: Vibration analysis and suppression control of an elevator string actuated by a PM synchronous servo motor, Journal of Sound and Vibration, 206, (1997), pp. 399-423.

(3) Otsuki M., Ushijima Y., Yoshida K., Kimura H., Nakagawa T.: Application of nonstationary sliding mode control to suppression of transverse vibration of elevator rope using input device with gaps, JSME International Journal, 49-2, C, (2006), pp.385-394.

(4) Okada, K., Sugiyama, M., Yamazaki, Y., Tomisawa, M., Vibration Control of Super-High-Speed Elevators (2nd Report, Study on Vibration Suppression Method Using An Actual System), Transactions of the Japan Society of Mechanical Engineers, 60-579, C, (1994), pp.3782-3788 (In Japanese).

(5) Yamazaki, Y., Tomisawa, M., Okada, K., Sugiyama, Y., Kojima, K., Saragai, K., Vibration Control of Super-High-Speed Elevators (1st Report, A Field test of Car Traveling), Transactions of the Japan Society of Mechanical Engineers, 61-581, C, (1995), pp.15-21 (In Japanese).

(6) Teshima, N., Kamimura, Nagai, M., Kou, S., Kamada, T., Vibration Control of Ultra High Speed Elevator by Active Mass Damper (1st report, Study by Optimal Control Theory), Transactions of the Japan Society of Mechanical Engineers, 65-637, C, (1999-9), pp.3479-3485 (In Japanese).

(7) Teshima, N., Kamimura, K., Hirai, M., Nagai, M., Kamada, T., Vibration Control of Ultra High Speed Elevator by Active Mass Damper (2nd report, Experiments Using a Full-Scale Test Facility), Transactions of the Japan Society of Mechanical Engineers, 66-647, C, (2000), pp.2181-2186 (In Japanese).

(8) Utsunomiya, K., Okamoto, K., Yumura, T., Sakuma, Y., Vibration Control of High-Speed Elevators Taking Account of Electricity Consumption Reduction, Transactions of the Japan Society of Mechanical Engineers, 72-719, C, (2006), pp.2048-2055 (In Japanese).

(9) Yamazaki Y., Tomisawa M., Okada K., Sugiyama Y.: Vibration control of super-high-speed elevators (Cabin vibration control method based on computer simulation and experiment using a full-size cabin model), JSME International Journal, C, 40-1, (1997), pp.74-81. 